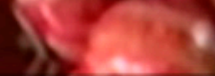

PUEBLL, CULTURA E IDENTDAD
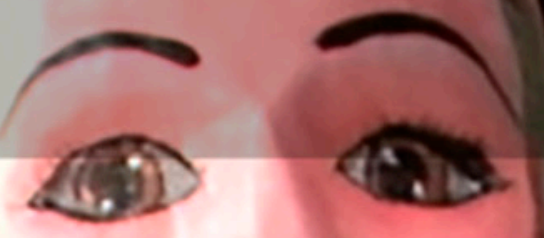

6 in

as ard

anisy $a^{2}$

1.
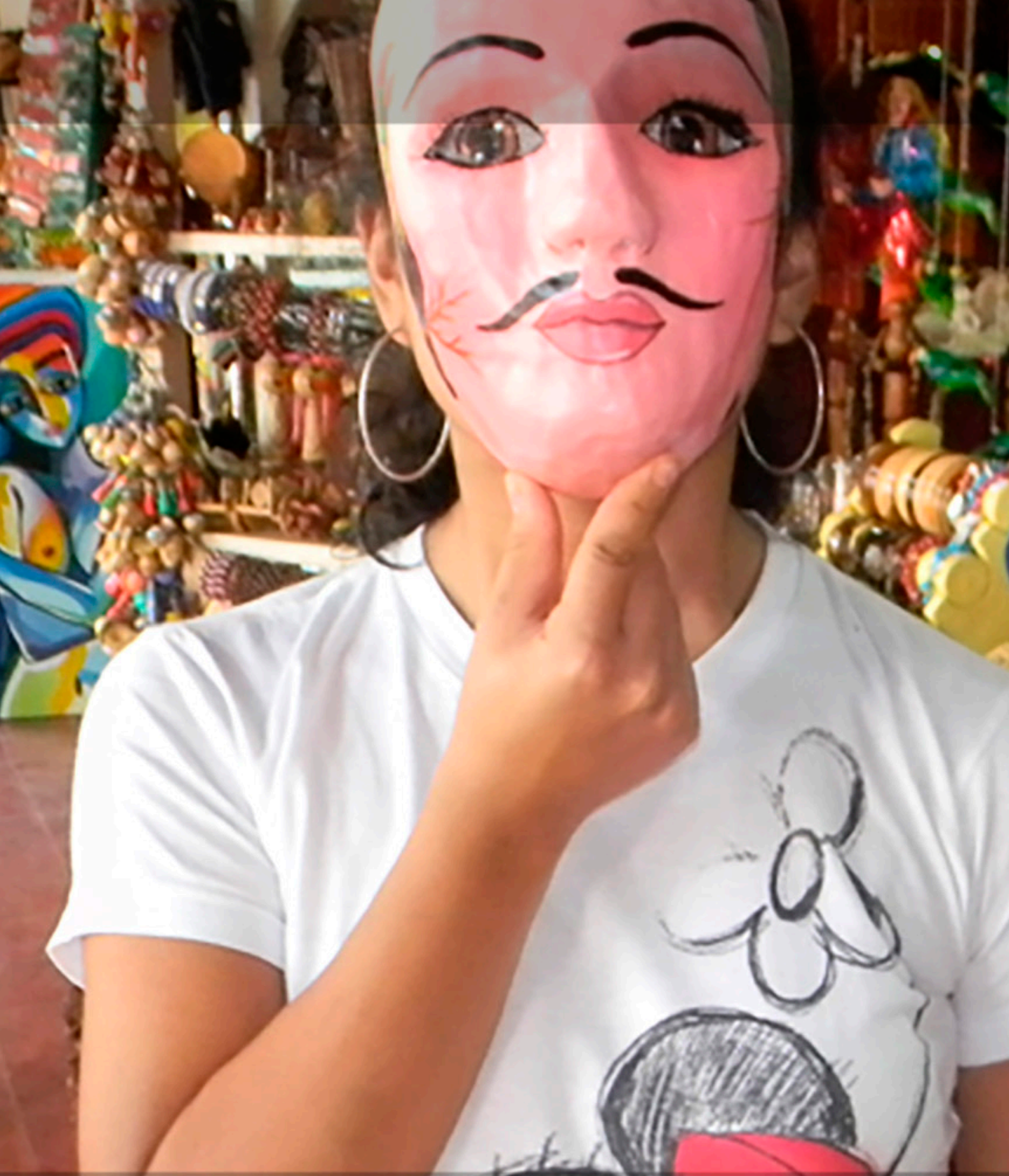

Una joven nacida en Estados Unidos visita el país de nacimiento de sus padres, Nicaragua, posando una máscara, las que han sido utilizada por sus antepasados para el baile y la fiesta. Símbolo de la herencia cultural. Son muchos los jóvenes de padres nicaragüenses nacidos en otras latitudes que poco a poco vuelven su vista a sus raices.

Foto: Xavier Rodriguez, 2011 


\section{Reconocimiento del liderazgo tácito de protagonistas claves en la comunidad indígena de Monimbó}

\section{Recognition of the unspoken leadership of key players in the indigenous community of Monimbó}

\section{Roberto Arana Vargas.}

Consultor JA Nicaragua - INDE

ID Orcid: https://orcid.org/0000-0003-1560-1151

bayron89@hotmail.com

\section{Resumen}

Un artículo que expone análisis y reflexiones generadas a partir de conceptos propuestos por el Profesor Luis Felipe Ulloa en su obra acerca del protagonismo y la construcción del futureo como una visión idealizada y desarrollada producto de la identificación de actores claves dentro de los diferentes ámbitos que conforman la dinámica de una comunidad. Estas bases teóricas han sido aplicadas a partir de la observación del trabajo de campo etnográfico en la comunidad indígena de Monimbo de la ciudad de Masaya en la que se propone una nueva conceptualización de "liderazgo tácito", anónimos lideres conectores que mueven todo un engranaje desde las raíces del sistema para su adecuada coordinación con todo un sistema establecido que se transforma día a día para producir una imagen que fortalezca el camino, y vivifique el hecho de ser indígenas, hoy en día en tiempos de la búsqueda del buen vivir.

Palabras clave: Liderazgo, pueblo, comunidad indígena, buen vivir, protagonismo, cultura, identidad

\section{Abstract}

An essay that exposes analysis and reflections generated from concepts proposed by Professor Luis Felipe Ulloa in his work about protagonism and the construction of the future as an idealized and developed vision product of the identification of key actors within the different areas that make up the dynamics of a community. These theoretical bases have been applied from the observation of the ethnographic field work in the indigenous community of Monimbo in the city of Masaya in which a new conceptualization of "tacit leadership" is proposed, anonymous connector leaders that move a whole gear from the roots of the system for its proper coordination with an established system that is transformed every day to produce an image that strengthens the way, and vivify the fact of being indigenous, today in times of the search for good living.

Keywords: Leadership, people, indigenous community, good living, prominence, culture, identity 


\section{Introducción}

En estas páginas se platea cómo en la investigación "Los ritos de la muerte y los procesos adaptativos del duelo" se realiza un análisis sobre la comunidad indígena de Monimbó en la ciudad de Masaya, considerando involucrar críticamente categorías y conceptos propuestos por Luis Felipe Ulloa F., en varias publicaciones y presentaciones. Se desarrolla este trabajo en los siguientes niveles: explorando, aplicando y posiblemente avanzando, lo que el autor mencionado ha denominado "ámbitos", "dinámicas" y "prácticas". En el caso de los tipos de protagonismos, personas y colectivos que marcan la vida cotidiana de una comunidad, se ha introducido en la tipificación de líderes, promotores (o impulsores) y ejecutores modelo. Y refiriéndose a horizontes, ha interesado la construcción colectiva e idealizada de la imagen de comunidad bajo el "método del futureo", que ha tomado forma con otros investigadores en Brasil.

Para dicho proceso se utilizó el método etnográfico tomando como unidad de análisis la dinámica de liderazgo comunitario triangulado con los fundamentos teóricos del Protagonismo y Futureo del Prof. Luis Felipe Ulloa. Las técnicas utilizadas para recolección de la información fueron: Diario de campo, observación directa y entrevista durante el periodo de noviembre 2017- noviembre 2018 en la comunidad indígena de Monimbo en la ciudad de Masaya, Nicaragua.

\section{Apuntes sobre Monimbó, barrio indígena de Masaya}

Se pretende considerar la aplicabilidad y ajuste, si fuese necesario, de dichas estrategias y realizar el análisis de la interacción entre las estructuras y sub estructuras presentes. Todo esto con el fin de generar una descripción dinámica, viva e incluso cambiante de las interrelaciones y rangos de influencia entre los diferentes actores.

Y el camino se va haciendo explorando elementos con el interés de identificar marcas identitarias y diferenciales que hacen a Monimbó única y distinta a los otros entornos y comunidades de la ciudad de Masaya. Así que es un buen momento, para ubicar al lector o lectora, haciendo un breve recorrido por sus calles y avenidas, por su gente y sus artesanías, por su cultura y sus creencias:

Se encuentra ubicada en la zona sur oeste de la ciudad de Masaya abarcando un límite geográfico de las cercanías

del parque

central hasta

la carretera

de Catarina-

Las Flores y el desfiladero de la laguna de Masaya. Más que un barrio en términos físicos o una ubicación geográfica resalta una vida propia, que basa su identidad en un vínculo ancestral con raíces chorotegas enriquecido con un pintoresco espíritu laborioso que plasma la vida cotidiana de un grupo étnico que ha habitado en este sector desde tiempos precolombinos.

En relación al abordaje del tema de investigación ya mencionado se ha buscado conocer sus percepciones de la vida y la muerte, sus creencias y cosmovisiones trascendentalistas, sus conexiones intergeneracionales y la permanencia de aspectos ancestrales que han logrado conservar a lo largo del tiempo.

Más que hacer una descripción de cómo lloran a sus muertos y cómo se adaptan en el proceso posterior a la partida y el duelo, se busca conocer su filosofía de vida, cómo convocan no solo la memoria de sus deudos sino también sus enseñanzas afirmando su identidad, así como establecer mejor relación entre esta práctica y otras dinámicas siempre interconectadas tales como la religión, la danza, la gastronomía, de las que no se puede dejar de reconocer el rol de su estructura de liderazgo autónoma y legítimamente reconocida por sus habitantes.

Estas reflexiones orientan a identificar el funcionamiento de su sistema en el cual se logró localizar no solo estructuras sociales, políticas, religiosas, económicas, recreativas sino también aquellas vinculadas a las raíces culturales. Algunas 
de estas surgen y se sostienen de aparente forma espontánea y casi natural, sin embargo, cuando se profundizan las observaciones se logra identificar que esos barcos de vela son impulsados por vientos de liderazgo a veces imperceptibles pero muy presentes que condicionan y dirigen no solo las actividades desarrolladas, sino también la transmisión de conocimientos, la asignación de roles, la permanencia del sistema inyectado de forma multidireccional tanto a sectores de contemporáneos como a las nuevas generaciones emergentes.

La familia y la comunidad son ámbitos muy conectados en la comunidad, de manera que algunas dinámicas no se entienden suficientemente si descartamos uno de estos dos ámbitos. En definitiva, la comunidad de Monimbó da gran relevancia a la organización familiar o vinculo sanguíneo. Muchos de los hogares mantienen estructura de familia ampliada regidas por los parientes de mayor edad que gozan de cierto respeto por el resto de los integrantes.

Muchas de las viviendas albergan a más de una familia en su interior, algunas tienen la característica de tener una sala con cuartos en la fachada exterior y al llegar al patio hay otras viviendas en el interior de la casa donde conviven hasta tres generaciones dentro de una misma propiedad. Estos nexos se mantienen no solo dentro de una misma propiedad, sino que también en aquellas relaciones de familias emparentadas entre sí. Estos mismos grupos familiares también son conocidas por sus apellidos tales como "Ambota, Namoyuri, Ticay o Ñurinda" y también por sus peculiares apodos como "los monos, los pata de chancho, los vizcos, los negros, los lambriscos". Alguien comentaba: "Si sos de Monimbo, tenés un apodo".

Cada familia es conocida también por las funciones que acostumbran a desempeñar, oficios tradicionales que en muchos casos son transmitidos de generación en generación. Algunas familias enteras se dedican a la elaboración de hamacas, otras a la talabartería, calzado, artesanías de bambú tales como canastos o muebles, juguetes tradicionales, adornos, accesorios típicos, cajetas, churritos, pintura, vestuario entre otros. Así que la dinámica económico-productiva, pasa a entenderse también como un elemento identitario familiar.
Es interesante que el aporte que ofrecen a nivel familiar es de una sola línea, es decir, si se dedican a la elaboración de alimentos tradicionales, solo eso replica el resto de los integrantes. Al no haber tanta diversificación en el producto que ofrecen puede ser un punto negativo cuando la demanda del mismo llega a disminuir afectando el único ingreso de toda una familia. No todos optan por darle continuidad al oficio familiar, las generaciones más jóvenes asisten a la universidad, algunos buscan carreras que les apoyen en la administración de los negocios familiares, pero otros optan por incursionar en empresas fuera de la comunidad.

\section{Liderazgo tácito en la consolidación cultural e identitaria}

En este análisis se ha ido activando un concepto que ya es producto de la investigación que se va desarrollando: La existencia de un liderazgo tácito de protagonistas claves en el buen vivir de la comunidad y también en la transmisión y consolidación cultural-identitaria con algunos elementos asociados a las observaciones de campo en la comunidad indígena de Monimbó.

Algunas de las inquietudes y vicisitudes que han expresado los líderes de las estructuras de ancianos y alcaldía de Vara tienen que ver con cómo llegar a las nuevas generaciones que aparentemente no mostraban mucho interés en el continuum de las tradiciones dejando entrever una brecha intergeneracional asediada por la globalización, prestamos e imposiciones culturales.

El tema de la autoidentificación étnica tomaba forma como una de las principales problemáticas para sus pobladores. Durante el proceso de trabajo de campo con mucha frecuencia los habitantes se identificaban así mismos como "monimboseños" pero al tener que identificarse como indígenas lo sentían de forma peyorativa y en algunos casos hasta se afrentaban de esta identidad o simplemente no la reconocían.

En el párrafo anterior escrito en tiempo pasado porque se considera que, con la crisis política reciente, este sentido de pertenecía fue revitalizado y el orgullo de sus miembros de una u otra forma fue impulsado fortaleciendo su cohesión interna como indígena. Muchas veces es a través de las crisis y dificultades cuando la familia se reúne y profundiza sus lazos de integración. 
El aspecto teórico de los protagonistas es clave en la descripción de la dinámica social de esta comunidad. Monimbo es tierra de artesanos, tierra de folclore, tierra de picardía y de tradiciones. Es esa la llama viva en su frente altiva y trabajadora la que les antecede en su fama. La esencia misma de su propia concepción y las inervaciones existentes entre las diversas estructuras no lograrían la eficiencia obtenida sin el apoyo de aquellos elementos humanos que se han adherido a la estructura organizativa comunitaria y que comparten una visión e identificación con la causa que les hace ser miembros o mantenerse dentro de ese sistema.

Población abundada por "lideres tácitos" presentes como conectores entre el sentir y la visión del grupo, como hilos que establecen conexión y permiten la influencia de quien coordina en una u otra dinámica, en uno u otro quehacer, facilitando la comunicación y vínculos que son fundamentales para el fortalecimiento de la adhesión grupal y a quienes se define como aquellos silenciosos pero relevantes miembros del engranaje que permiten la marcha del "todo".

Este concepto ha surgido como resultado de las observaciones in situs. Aplicando este fenómeno al entorno donde se ha realizado las consideraciones surgen las preguntas ¿Qué los mantiene unidos? ¿Cuáles son aquellos elementos que vinculan al grupo y les permite cohesionarse y continuar conectados? Estos marcadores son de gran relevancia para posteriormente ubicar los puntos de convergencia dentro de la gran estructura comunitaria que en muchos casos pueden ampliar su influencia a más de una esfera organizativa, e incluso a otros espacios, ámbitos y territorios.

Tomar en cuenta la estructura organizativa familiar y el tipo de actividad económica, permitió ubicar a líderes focales o conectores de estructuras que en el caso de las que son familiares usualmente son los más ancianos quienes en la opinión del suscrito autor de este manuscrito y con base a lo que ha logrado observar, gozan de gran respeto y son vistos como consejeros manteniendo un contacto cercano con todos los integrantes de la familia que solamente en muy pocas ocasiones se van a vivir distantes de sus casas maternas.

En el caso del liderazgo económico, generalmente se organiza conforme al tipo de actividad o función económica estructurada desde los negocios que han logrado monopolizar el tipo de servicio ofrecido hasta los artesanos con menor cantidad de producción. Algunos se han organizado en cooperativas artesanales que aún pueden ser potencializadas en gran manera con el objetivo de mejorar la calidad de la producción y fortaleciendo así la economía familiar y ayudándoles en la gestión de la distribución del producto y la identificación de nuevos mercados en el exterior.

Esta área económica tiene sub estructuras también asociadas por producto "las fritangueras, los panaderos, los carpinteros, los pintores, los zapateros, los cajeteros, los que elaboran fuegos artificiales o trabajan con pólvora, los campesinos, los marimberos, los costureros, los que crían animales, las mercaderas". No todos cuentan con estructuras organizativas formales, se asocian entre sí, se conocen, cuentan con personas de referencia que en ciertas ocasiones alzan la voz en nombre del grupo, pero su funcionamiento se da en un continuum casi sincronizado por reglas dictadas al aire.

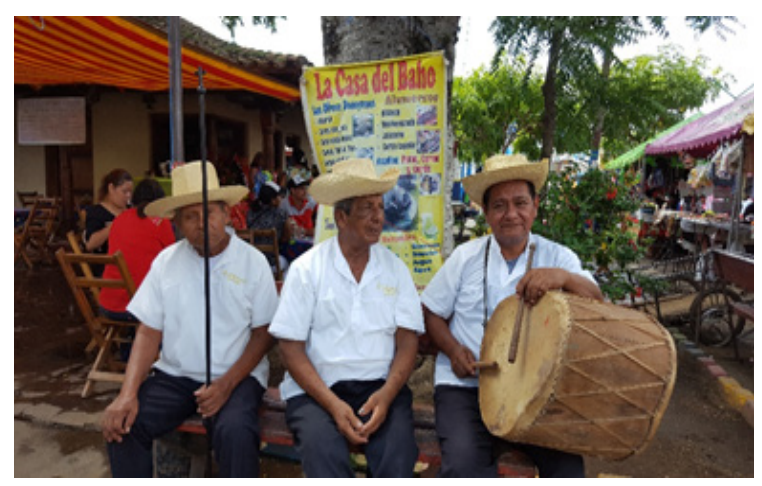

Luego se encuentra otra estructura muy importante y es la de las cofradías, grupos culturales y tradicionales que son una parte elemental de la marca identitaria de la comunidad, son quienes transmiten de generación a generación las prácticas y creencias que son un sello muy importante para el monimboseño. En este punto se encontraron grupos familiares y grupos mixtos organizados e integrados por los que ejecutan instrumentos familiares: "los chicheros, los marimberos, los guitarristas", los que bailan música tradicional organizados en grupos folclóricos que a veces son compuestos por miembros de una sola familia y en otras ocasiones por el tipo de baile que interpretan, "los de negra, los tradicionales, del toro 
venado, de los diablitos entre otros". En este punto también surgen lideres culturales, personas que se convierten en referencia y puntos clave para el desarrollo cultural de la comunidad de Monimbó.

Son voces respetadas que brindan instrucciones y orientan a los más jóvenes y participan activamente en las diferentes celebraciones culturales y religiosas, que por cierto son perennes y muy prolongadas en casi todo el año.

Desprendido de este punto cultural y tradicional se ha logrado identificar una estructura de liderazgo formal y ampliamente reconocida por los monimboseños y es el consejo de ancianos y la alcaldía de vara quienes son considerados los heraldo y portadores del patrimonio identitario y gozan de una posición de respeto y cierta obediencia ante algunas actividades tales como la roza de los panteones que se realiza 4 veces al año, alguna reuniones con el liderazgo de la comunidad, situaciones de emergencia o desastres.

Ante estas eventualidades, los sujetos son convocados por un "titante" que suena un tambor de madera de aguacate, antiguamente de piel de venado quien recorre la localidad para llamarles al punto de reunión designado. Ellos se reúnen a la sombra de sus "teites" para honrar las memorias vivas de sus ancestros.

No por nada Monimbó es considerado el corazón de Masaya de la cual Darío expresó en su poema "Gratitud a Masaya":

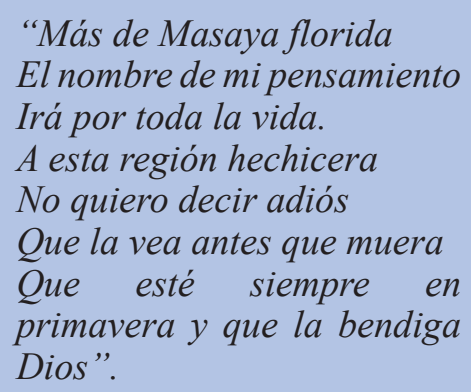

Este gran valor cultural e intangible puede fungir como un hermoso semillero que sea aún más diseminado en la proyección de la idiosincrasia tan diversa de Nicaragua y fuera de ella.

El buen funcionamiento de un sistema y la sobrevivencia del mismo dependerán en gran manera de sus formas y expresiones de organización y funciones de liderazgo que provean orden y protección a sus integrantes. Estas estructuras no podrían ser funcionales sin el roll que juegan sus conectores y representantes que surge de aparente forma espontánea y que a pesar de no ser tan notorios se convierten en brazos e hilos que vigorizan la efectividad y cumplimiento de asignaciones de sus integrantes. Se confirma que ninguna dinámica social está disociada de otra, que la danza se relaciona a la música y ambas a las artesanías, la gastronomía a las fiestas y las fiestas a la religión, todas ellas tienen exponentes o referentes claves, protagonistas que engranan y mueven el medio. Estos a la vez actúan, se comportan acorde a las características e idiosincrasias particulares del entorno cultural donde se desarrollan.

\section{Conclusión}

Como resultado de la relación con la comunidad, se puede inferir que ella podría jugar en el futuro un papel, como una tierra de armoniosa coordinación que ha aprendido a través de la experiencia del tiempo a mantenerse unida pese a los abruptos cambios del exterior luchando por preservar su esencia y su llama viva de tradiciones y creencias en lo que el liderazgo asume un roll importante en el funcionamiento de este hermoso, simple y complejo engranaje comunitario arraigado a una sangre rebelde, orgullosa, alegre y laboriosa, una sangre chorotega, una sangre monimboseña.

Una comunidad suspendida entre su pasado y su futuro, que lucha por sobrevivir adaptándose, creciendo, aprendiendo y fortaleciendo sus lazos internos de unidad familiar. Sin embargo, es un ejercicio de "futureo", que ha de ser impulsado entre los distintos sectores de la comunidad, para producir una imagen que fortalezca el camino, y vivifique el hecho de ser indígenas, hoy en día en tiempos de la búsqueda del buen vivir.

\section{Bibliografía.}

Ulloa, L. F. (2009). Protagonismo desde adelante, desde atrás, desde todas partes. Fondo editorial.

Ulloa, L. F. (2010). Siete Pláticas. Puerta Abierta. 
Roberto Arana Vargas.

Psicólogo clínico egresado con honores de la facultad de Ciencias médicas de la Universidad Cristiana Autónoma de Nicaragua, UCAN. Cuenta con estudios de posgrado en psicología forense de la Universidad Martin Lutero y con práctica en el campo de la psicología educativa y clínica. Posee estudios en salud sexual y reproductiva y Derechos Humanos del Centro de Investigación de Estudios Superiores, CIES, también tiene estudios de teología en el Training Pastoral Institute y un diplomado en liderazgo del American College. Actualmente es maestrante en antropología y liderazgo social en la Universidad Nacional Autónoma de Nicaragua, UNAN.

Es miembro de la red latinoamericana de risoterapia en la cual es risoterapeuta certificado, ha sido consultor en temas de desarrollo comunitario, liderazgo juvenil, emprendimiento empresarial, participación ciudadana y orador en temas de desarrollo personal y motivación en el Instituto nicaragüense de desarrollo, INDE, y Emprendedores Juveniles de Nicaragua entre otras. Ha sido docente en el Instituto del Pacifico de Chosen Children, facilitador en programas de Junior Achievement Nicaragua y expositor en distintas instituciones de educación superior. Actualmente es el presidente del Instituto Nicaragüense de Cultura Hispánica, filial Masaya, y coordina a nivel nacional la red de voluntarios de JA Nicaragua. 